\title{
Effect of ORF7 of SARS-CoV-2 on the Chemotaxis of Monocytes and Neutrophils In Vitro
}

\author{
Gang Wang $\mathbb{D}^{1},{ }^{1}$ Jun Guan, ${ }^{1}$ Guojun Li, ${ }^{2}$ Fengtian Wu, ${ }^{1}$ Qin Yang, ${ }^{1}$ Chunhong Huang, \\ Junwei Shao, ${ }^{1}$ Lichen Xu, ${ }^{1}$ Zixuan Guo, ${ }^{1}$ Qihui Zhou, ${ }^{1}$ Haihong Zhu $\mathbb{D}^{1},{ }^{1}$ and Zhi Chen $\mathbb{B D}^{1}$ \\ ${ }^{1}$ State Key Laboratory for Diagnosis and Treatment of Infectious Diseases, National Clinical Research Center for Infectious Diseases, \\ Collaborative Innovation Center for Diagnosis and Treatment of Infectious Diseases, The First Affiliated Hospital, \\ Zhejiang University School of Medicine, Hangzhou, China \\ ${ }^{2}$ Department of Liver Diseases, National Clinical Research Center for Infectious Disease, Shenzhen Third People's Hospital, \\ The Second Affiliated Hospital, School of Medicine, Southern University of Science and Technology, Shenzhen, \\ 518000 Guangdong, China
}

Correspondence should be addressed to Haihong Zhu; zhuhh72@zju.edu.cn and Zhi Chen; zjuchenzhi@zju.edu.cn

Received 15 July 2021; Revised 29 August 2021; Accepted 13 September 2021; Published 28 September 2021

Academic Editor: Wen-Jun Tu

Copyright (C) 2021 Gang Wang et al. This is an open access article distributed under the Creative Commons Attribution License, which permits unrestricted use, distribution, and reproduction in any medium, provided the original work is properly cited.

Coronavirus disease 2019 (COVID-19) caused by the severe acute respiratory syndrome coronavirus 2 (SARS-CoV-2) is currently the most significant public health threat worldwide. Patients with severe COVID-19 usually have pneumonia concomitant with local inflammation and sometimes a cytokine storm. Specific components of the SARS-CoV-2 virus trigger lung inflammation, and recruitment of immune cells to the lungs exacerbates this process, although much remains unknown about the pathogenesis of COVID-19. Our study of lung type II pneumocyte cells (A549) demonstrated that ORF7, an open reading frame (ORF) in the genome of SARS-CoV-2, induced the production of CCL2, a chemokine that promotes the chemotaxis of monocytes, and decreased the expression of IL-8, a chemokine that recruits neutrophils. A549 cells also had an increased level of IL-6. The results of our chemotaxis Transwell assay suggested that ORF7 augmented monocyte infiltration and reduced the number of neutrophils. We conclude that the ORF7 of SARS-CoV-2 may have specific effects on the immunological changes in tissues after infection. These results suggest that the functions of other ORFs of SARS-CoV-2 should also be comprehensively examined.

\section{Introduction}

Coronavirus disease 2019 (COVID-19) is an infectious disease caused by the severe acute respiratory syndrome coronavirus 2 (SARS-CoV-2) that has become a worldwide pandemic [1-3]. The number of confirmed patients infected by SARS-CoV-2 continues to increase daily. As of Apr 2021, there were more than 0.14 billion SARS-CoV-2 infections and 3 million deaths from COVID-19 reported worldwide (https://coronavirus.jhu.edu/map.html). This global pandemic is still not under control, although there are encouraging trends in some regions. Other coronaviruses, such as MERS and SARS, had high transmissibility, but the epidemics were limited to certain regions and populations.
Thus, SARS virus led to more than 8000 infected cases and 700 deaths in 26 countries, and MERS led to about 2500 cases and 858 deaths in 27 countries [4-6]. In contrast, there has been an enormous disease burden associated with SARS$\mathrm{CoV}-2$ infection. Numerous vaccines are currently available in many regions, and clinical trials have shown they are effective and safe $[7,8]$.

SARS-CoV-2 infects lung epithelial cells, type II alveolar (ATII) cells, by binding to the membrane-associated angiotensin-converting enzyme 2 (ACE2) on the cell surface [9-12]. Once inside the host cell, SARS-CoV-2 begins to produce viral RNA polymerase, which then replicates the complementary genomic RNA, making double-stranded RNA [13]. Subsequently, cells translate the structural and 
nonstructural proteins (NSPs) of SARS-CoV-2 in the cytosol $[13,14]$. The structural proteins include nucleocapsid $(\mathrm{N})$, spike (S), membrane (M), and envelope (E) proteins, and the NSPs include 16 NSPs from the ORF1ab [15]. There are at least 10 open reading frames (ORFs) in the genome of SARS-CoV-2: ORF1ab, ORF2 (S protein), ORF3, ORF4 (E protein), ORF5 (M protein), ORF6, ORF7, ORF8, ORF9 (N protein), and ORF10 $[15,16]$. Viral polymerase and all 16 NSPs are translated from the ORF1ab subgenome [16]. The S, N, E, and $\mathrm{M}$ proteins are all structural proteins, and the 16 NSPs function in replication and transcription of the viral genome [15].

Emerging evidence indicates that almost all of these ORF proteins have important roles in the lifecycle of SARS-CoV2. As an RNA virus, SARS-CoV-2 infection stimulates the innate immunity of cells for RNA sensor proteins in the cytosol, such as retinoic acid inducible gene-I (RIG-I), melanoma differentiation-associated gene-5 (MDA-5), and toll-like receptors (TLR 3/7/8), which induce the expression of interferons (IFNs) [17]. ORF6, ORF8, and the N protein of SARS-CoV-2 inhibit these IFN-activated antiviral pathways, and this inhibits the IFN-stimulated response element (ISRE) [18]. Additionally, ORF3 upregulates markers of apoptosis in 293T, HepG2, and Vero E6 [19]. Although the functions of several ORFs are incompletely understood, all ORFs and NSPs have specific functions during the lifecycle of SARS-CoV-2.

The present in vitro study examined the function of ORF7 in SARS-CoV-2 by focusing on its regulation of numerous cytokines and chemokines (IL-6, TNF-a, IL-8, CXCL2, and CXCL7) that function in the chemotaxis of monocytes and neutrophils in vitro.

\section{Materials and Methods}

2.1. Cell Culture and Vector Construction of ORF7. A lung adenocarcinoma cell line (A549) was purchased from the Chinese Academy of Science (Shanghai, China), and cells were cultured in Dulbecco's modified Eagle medium (DMEM; Hyclone, Logan, UT, USA) with $10 \%$ fetal bovine serum (FBS; Corning, NY, USA) and 1\% penicillin/streptomycin in a humidified incubator with $5 \% \mathrm{CO}_{2}$ at $37^{\circ} \mathrm{C}$. For transfection, a lentiviral vector harboring FLAG-tagged ORF7 of SARS-CoV-2 was constructed using a specific SARS-CoV-2 strain (Wuhan-Hu-1 strain, NC_045512). Transfection was performed, and ORF7-expressing A549 cell was obtained. Control cells were transfected with control vector.

2.2. Quantitative Real-Time Polymerase Chain Reaction ( $q R T-P C R)$. RNA was extracted from cells using the TRIzol reagent (TaKaRa, Dalian) and was then reverse transcribed into cDNA using the PrimeScript RT Master Mix (TaKaRa, Dalian). The expression of mRNAs (IL-1a,IL-1b, IFN- $\alpha$, $I F N-\beta, I L-6, I L-8, C C L 2$, and $T N F-\alpha)$ was quantified using qRT-PCR with the TB Green Master Mix (TaKaRa, Japan). Expression was normalized to $G A P D H$, and relative expression was calculated using the $2^{-\Delta \Delta \mathrm{Ct}}$ method (primers are listed in Table 1). qRT-PCR was performed with the Quant-
TABle 1: Primers for real-time PCR

\begin{tabular}{lc}
\hline Gene symbol & Sequences \\
\hline Human-3552-IL-1a-F & TGGTAGTAGCAACCAACGGGA \\
Human-3552-IL-1a-R & ACTTTGATTGAGGGCGTCATTC \\
Human-3553-IL-1b-F & AGCTACGAATCTCCGACCAC \\
Human-3553-IL-1b-R & CGTTATCCCATGTGTCGAAGAA \\
Human-3439-IFN-a-F & GCCTCGCCCTTTGCTTTACT \\
Human-3439-IFN-a-R & CTGTGGGTCTCAGGGAGATCA \\
Human-3456-IFN-b-F & GCTTGGATTCCTACAAAGAAGCA \\
Human-3456-IFN-b-R & ATAGATGGTCAATGCGGCGTC \\
Human-3569-IL-6-F & ACTCACCTCTTCAGAACGAATTG \\
Human-3569-IL-6-R & CCATCTTTGGAAGGTTCAGGTTG \\
Human-3576-IL-8-F & TTTTGCCAAGGAGTGCTAAAGA \\
Human-3576-IL-8-R & AACCCTCTGCACCCAGTTTTC \\
Human-7124-TNF-F & CCTCTCTCTAATCAGCCCTCTG \\
Human-7124-TNF-R & GAGGACCTGGGAGTAGATGAG \\
Human-6347-CCL2-F & CAGCCAGATGCAATCAATGCC \\
Human-6347-CCL2-R & TGGAATCCTGAACCCACTTCT \\
Human-6354-CCL7-F & GACAAGAAAACCCAAACTCCAAAG \\
Human-6354-CCL7-R & TCAAAACCCACCAAAATCCA \\
Human-3586-IL-10-F & TCAAGGCGCATGTGAACTCC \\
Human-3586-IL-10-R & GATGTCAAACTCACTCATGGCT \\
\hline
\end{tabular}

Studio $^{\mathrm{TM}}$ Dx system (ABI, Thermo, USA) using the following procedure: denaturation at $95^{\circ} \mathrm{C}$ for $5 \mathrm{~min}, 40$ cycles of $95^{\circ} \mathrm{C}$ for $5 \mathrm{~s}$ and $60^{\circ} \mathrm{C}$ for $30 \mathrm{~s}$, followed by a melting curve step of $95^{\circ} \mathrm{C}$ for $15 \mathrm{~s}$ and $60^{\circ} \mathrm{C}$ for $1 \mathrm{~min}$, and a final increase to $95^{\circ} \mathrm{C}$.

2.3. Immunofluorescence. ORF7-expressing A549 cells were cultured in 6-well plates with slides. After $24 \mathrm{~h}$, when the cells were adhered to coverslips, cells were fixed with $4 \%$ paraformaldehyde for $15 \mathrm{~min}$ and permeabilized with $0.5 \%$ TritonX-100. After blocking for $30 \mathrm{~min}$ at room temperature using 3\% BSA, the cells were incubated with a mouse antiFLAG antibody (Sigma, USA) at $4^{\circ} \mathrm{C}$ overnight and were then stained with a fluorescein isothiocyanate- (FITC-) conjugated goat anti-mouse antibody (ProteinTech) at room temperature for $1 \mathrm{~h}$. The nuclei were stained with DAPI (Abcam, ab104139), and the cellular distribution of ORF7 was observed using confocal microscopy.

2.4. Western Blot Analysis. Western blotting was conducted as previously described [20]. Briefly, cells were lysed with SDS sample buffer (1x), boiled for $10 \mathrm{~min}$, separated using 4-20\% SDS-PAGE (GenScript, USA), and then transferred onto a $0.22 \mu \mathrm{m}$ polyvinylidene difluoride (PVDF) membrane (Millipore, USA). After blocking for $1 \mathrm{~h}$ at room temperature with $3 \%$ BSA, the membranes were incubated with diluted primary antibodies at $4^{\circ} \mathrm{C}$ overnight. The secondary antibodies were added at room temperature for $2 \mathrm{~h}$. Protein bands were detected using the Clarity Western ECL Substrate (Bio-Rad, USA).

2.5. Isolation of Human Neutrophils and Monocytes. Neutrophils were isolated from blood samples of healthy human 


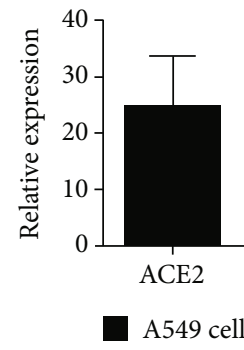

(a)

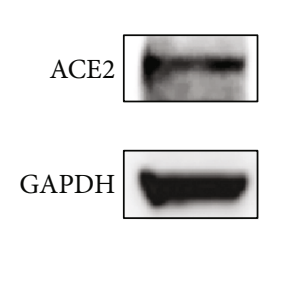

(b)
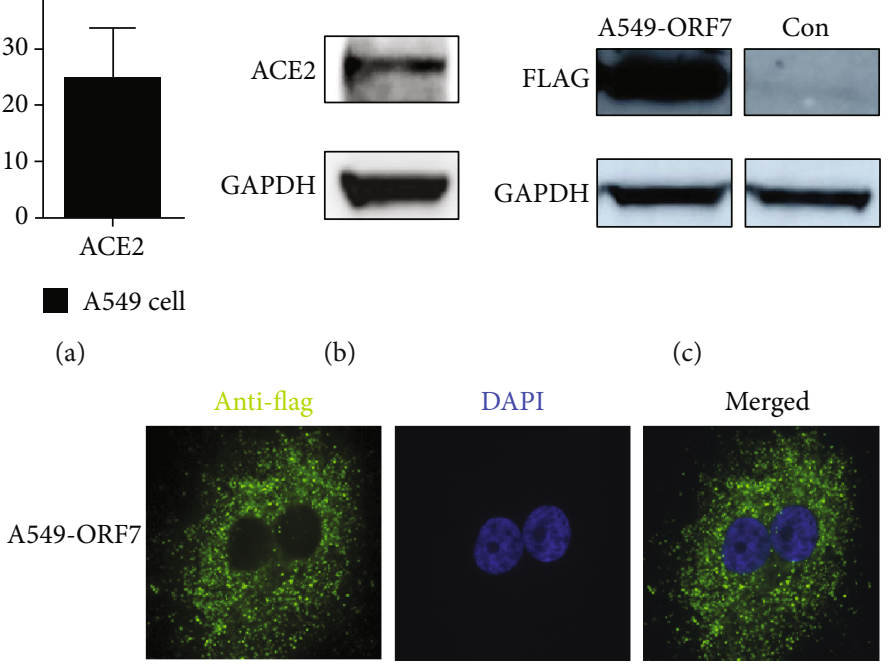

(c)
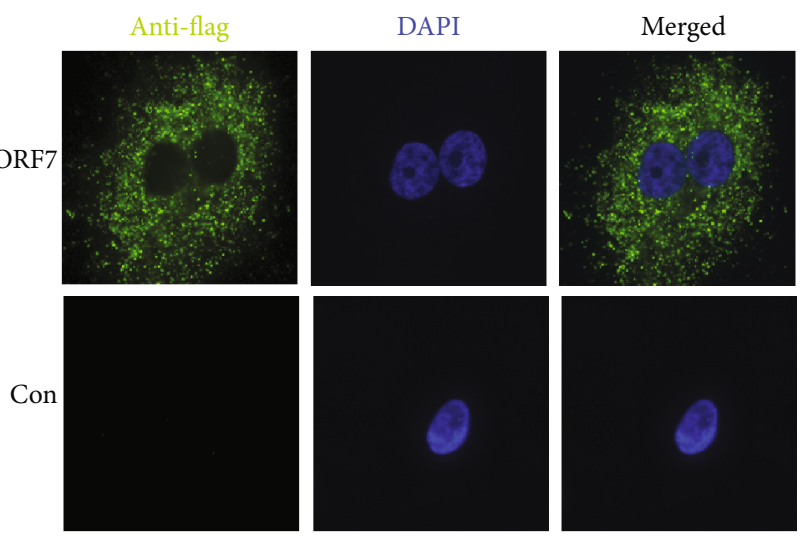

(d)

Figure 1: Expression of ACE2 and distribution of ORF7 in A549 cells. (a) Expression of ACE2 in A549 cells (qRT-PCR). (b) Expression of ACE2 in A549 cells (Western blotting). (c) FLAG-ORF7 expression in A549-ORF7 cells (Western blotting). (d) Cellular distribution of ORF7 in A549-ORF7 cells (immunofluorescence microscopy).

donors using PolymorphPrep (Alere Technologies AS, Oslo, Norway) as previously described $[21,22]$. Briefly, $5 \mathrm{~mL}$ of blood was layered onto $5 \mathrm{~mL}$ of PolymorphPrep and centrifuged for $35 \mathrm{~min}(500 \mathrm{~g}$ ) at room temperature. The neutrophil layer was transferred to a new tube, washed with PBS, diluted by $50 \%$ with $\mathrm{ddH}_{2} \mathrm{O}$, and then centrifuged for $10 \mathrm{~min}(400 \mathrm{~g})$, followed by red blood cell lysis in a lysis buffer (Solarbio, China).

Peripheral blood mononuclear cells (PBMCs) were isolated from blood samples of healthy donors using a Ficoll density gradient [23]. Then, CD14 microbeads (Miltenyi Biotec, Germany) were used for the positive selection of human monocytes from these cells [24]. The purity of the CD $14^{+}$cells was evaluated using an APC-conjugated antihuman CD14 antibody (eBioscience, CatNo: 17-0149-42) with flow cytometry. The Institutional Ethics Committee of the First Affiliated Hospital of Zhejiang University approved this study.

2.6. Transwell Assay. Transwell assays were conducted using a $12 \mathrm{~mm}$ Transwell with a $3.0 \mu \mathrm{m}$ pore polycarbonate membrane insert (Corning, USA, CatNo: CLS3402) [25]. A549 cells that were transfected with lentiviruses were seeded in the lower chamber and cultured for $24 \mathrm{~h}$ in DMEM containing $10 \%$ FBS. Human-derived monocytes and neutrophils in serum-free DMEM were added to the upper chamber. After incubation for $1 \mathrm{~h}$, cells in the reverse side of the upper chamber were fixed with $4 \%$ paraformaldehyde and then stained in crystal violet for observation with a microscope.
Cells in the lower chamber were collected and counted by flow cytometry, and counting beads were used for quantitation of different samples [26].

2.7. Enzyme-Linked Immunosorbent Assay (ELISA). ORF7expressing A549 cells and control cells were plated into 6well plates. The supernatant was collected and used for measurement of CCL2 (MultiSciences, CatNo: 70-EK187-96), CCL7 (Cloud-Clone Corp., CatNo: SEA089Hu), and IL-6 (MultiSciences, CatNo: 70-EK206/3-96) using ELISA kits according to each manufacturer's instructions.

2.8. Statistical Analysis. The significance of differences was determined using Student's $t$-test with GraphPad Prism version 7.0 (GraphPad Software, CA). A $P$ value below 0.05 was considered significant.

\section{Results}

3.1. Construction of ORF7-Expressing A549 Cells. ACE2 occurs on the surface of pneumocytes and binds to SARS-CoV-2 during infections [27]. We therefore first examined the expression of ACE2 in A549 cells, a type II pneumocyte cell line [28]. The Western blotting and quantitative PCR results confirmed that these cells expressed ACE2 (Figures 1(a) and 1(b)). We then used the sequence of a SARS-CoV-2 isolate (Wuhan-Hu-1, NC_045512.2) to construct a lentiviral vector that expressed a FLAG-tagged ORF7 subgenomic sequence (Lenti-ORF7-FLAG) and 


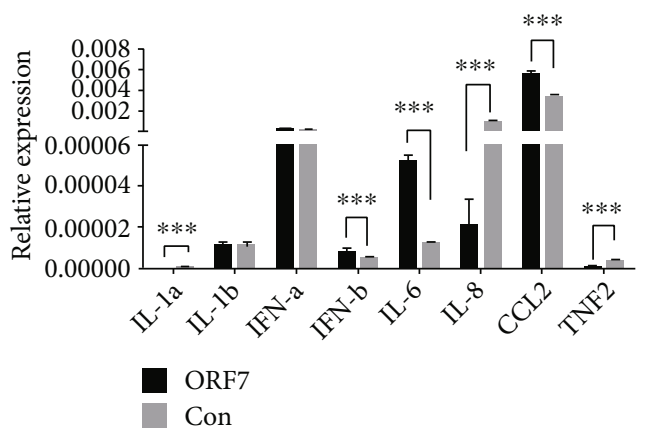

(a)

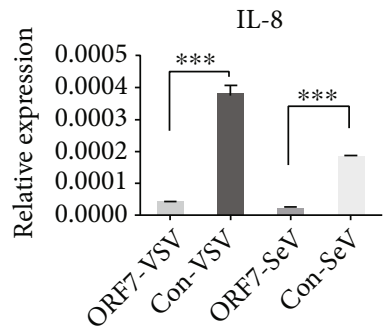

(c)

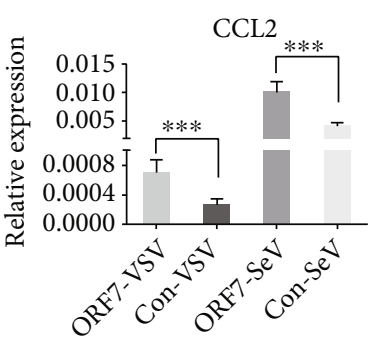

(b)

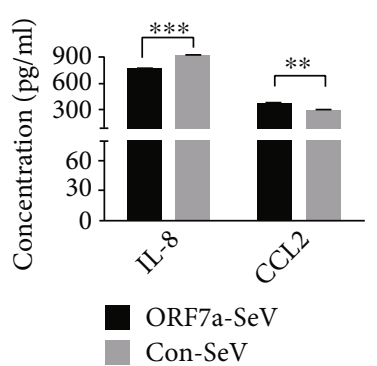

(d)

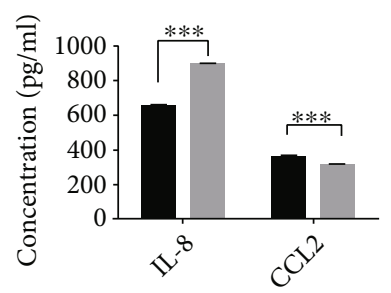

(e)

FIGURE 2: Effect of ORF7 on the expression of cytokines and chemokines in A549 cells. (a) Relative to control cells, A549-ORF7 cells had increased expression of $I L-6, C C L 2$, and $I F N-\beta$ (all $P<0.01$ ), decreased expression of $I L-1 \alpha, I L-8$, and TNF- $\alpha$ (all $P<0.01$ ), but similar expression of $I L-1 \beta$ and $I F N-\alpha$ (both $P>0.05$ ). CCL7 and $I L-10$ were undetectable (qRT-PCR). (b) Activation of innate immunity by $\mathrm{SeV} / \mathrm{VSV}$ infection increased the expression of CCL2 and decreased the expression of IL-8 in A549-ORF7 cells compared to control cells (both $P<0.05$; qRT-PCR). (c) A549-ORF7 cells had increased levels of CCL2 and decreased levels of IL-8 relative to control cells (both $P<0.01 ;$ ELISA).

transfected A549 cells with Lenti-ORF7-FLAG to establish an ORF7-expressing cells. We confirmed the expression and the cellular distribution of ORF7 using Western blotting and immunofluorescence. The results indicated expression of ORF7 (Figure 1(c)) and that this protein was present in the cytosol (Figure 1(d)). These data thus demonstrated the successful establishment of ORF7-expressing A549 cells that could be used for further studies of the function of ORF7.

3.2. ORF7 Alters the Expression of Cytokines and Chemokines in A549 Cells. Innate immune cells, such as monocytes, macrophages, and neutrophils, are the first cells to respond when there is an infection in the lungs [29]. In particular, infection of pneumocytes leads to massive infiltration of monocytes into lung tissues [30], although there appears to be limited infiltration of neutrophils during SARS-CoV-2 infection [31-33]. During the early stage of viral infection, cytokines (IL-1, IL-2, IL-8, IL-10, CCL2, CCL7, IFN- $\alpha$, IFN- $\beta$, and TNF- $\alpha$ ) have essential functions in the recruit- ment of immune cells, defense against the infection, and promotion of inflammation [34]. We therefore used qPCR to determine the expression of cytokines and chemokines in ORF7-expressing A549 cells compared to control cells (Figure 2(a)). The results demonstrated that IL-6, CCL2, and IFN- $\beta$ had higher expression in A549-ORF7 cells (all $P<0.01) ; I L-1 \alpha, I L-8$, and TNF- $\alpha$ had lower expression in A549-ORF7 cells (all $P<0.01$ ); the two groups had no differences in the levels of $I L-1-\beta$ and $I F N-\alpha$ (both $P>0.05$ ); and CCL7 and $I L-10$ were undetectable.

CCL-2 (MCP-1) and IL-8 (CXCL8) function in the chemotaxis of monocytes and neutrophils, respectively $[35,36]$. Sendai virus $(\mathrm{SeV})$ and vesicular stomatitis virus (VSV) infections stimulate intracellular innate immunity and can be used to model RNA virus infections [37]. We therefore used qPCR to measure CCL2 and $I L-8$ expression in A549ORF7 and control cells following infection by these viruses (Figure 2(b)). The results indicated that A549-ORF7 cells had greater expression of CCL2 and decreased expression 


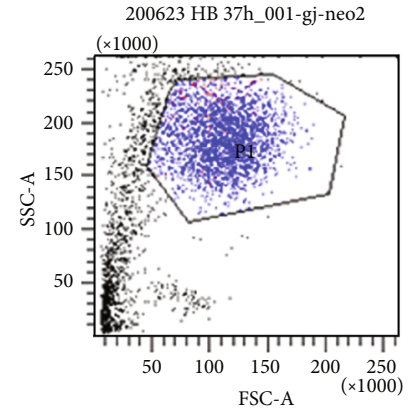

(a)

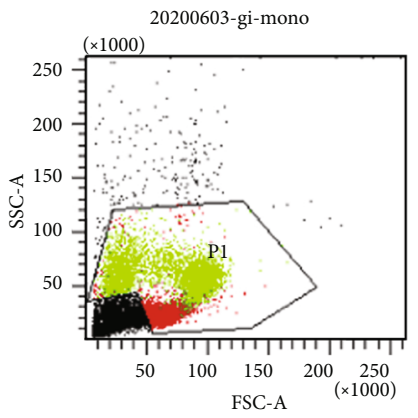

(c)

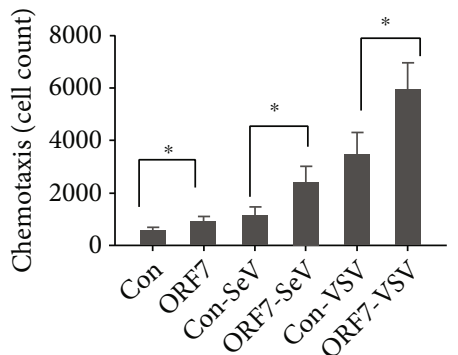

Monocyte

(e)

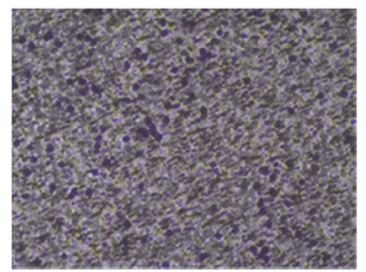

(g)

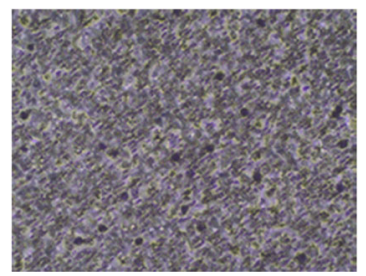

(i)

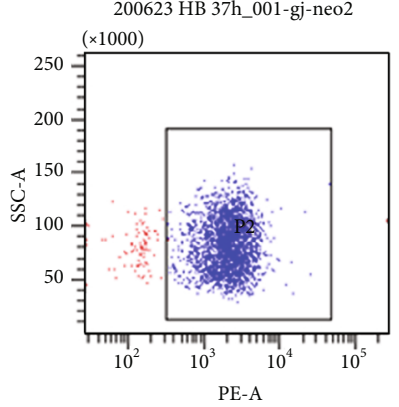

(b)

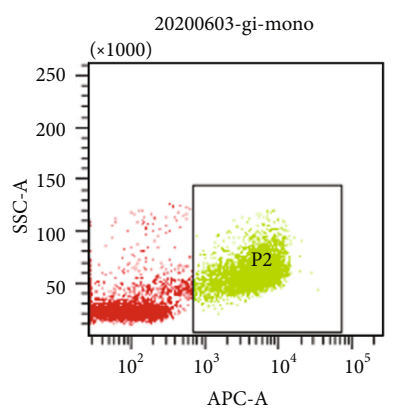

(d)

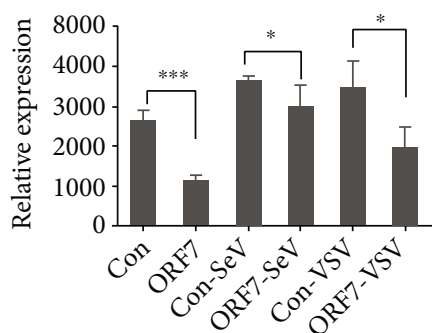

Neutrophil

(f)

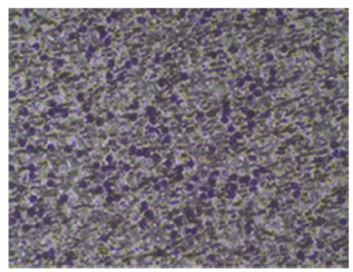

(h)

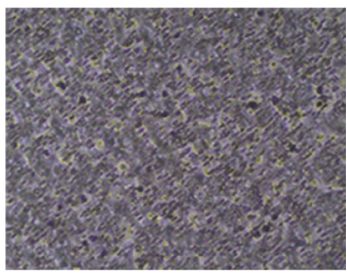

(j)

FIgURE 3: Continued. 


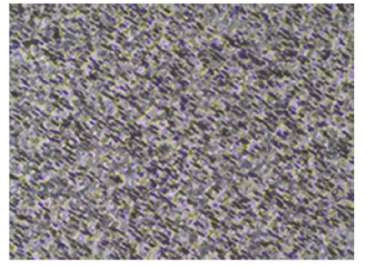

$(\mathrm{k})$

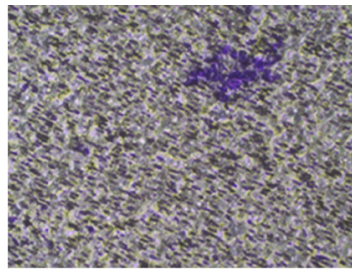

(m)

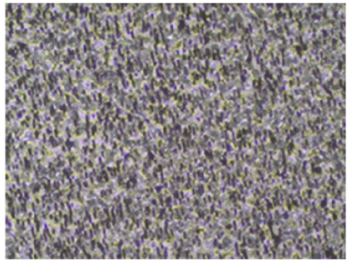

(1)

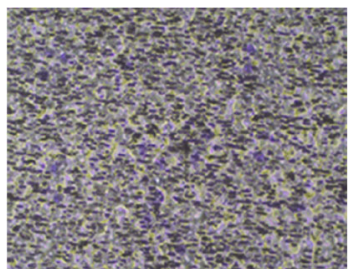

(n)

FIGURE 3: Effect of ORF7 on chemotaxis of neutrophils and monocytes. (a-d) Neutrophils were identified as CD11b+cells and monocytes as $\mathrm{CD} 14+$ positive cells (flow cytometry). ( $\mathrm{g}-\mathrm{n}$ ) Chemotaxis of monocytes and neutrophils (upper chamber) in response to A549-ORF cells (lower chamber) was examined by staining (Transwell assay). (e, f) Quantitation of chemotaxis results showing increased monocyte chemotaxis and decreased neutrophil chemotaxis (both $P<0.01$ ).

of $I L-8$ compared to control cells (both $P<0.05$ ). We also used ELISA to measure the levels of IL- 8 and CCL2 in the supernatant of A549-ORF7 and control cells (Figure 2(c)). These results confirmed that A549-ORF7 cells had increased expression of CCL2 and decreased expression of IL- 8 with or without infection by SeV/VSV or the transduction of agonist, LMW and HMW RNAs (both $P<0.01$ ).

3.3. ORF7 Affects the Chemotaxis of Monocytes and Neutrophils. We next examined the effects of ORF7 on the chemotaxis of monocytes and neutrophils. Flow cytometry provided identification of neutrophils as $\mathrm{CD} 11 \mathrm{~b}+$ cells (Figures 3(a) and 3(b)) and monocytes as CD14+ positive cells (Figures 3(c) and 3(d)). Next, we implanted the A549ORF7 and control cells in the lower chambers of 12-well plates, added monocytes and neutrophils in the upper chamber, and recorded chemotaxis after $6 \mathrm{~h}$ (monocytes) and $3 \mathrm{~h}$ (neutrophils) $[25,38]$. The results indicated that monocytes had increased chemotaxis, and neutrophils had reduced chemotaxis (Figures $3(\mathrm{~g})-3(\mathrm{n})$ ).

During these experiments, we found numerous transmembrane cells, monocytes, and neutrophils on the lower wells with the A549-ORF7 cells. Thus, we used flow cytometry for the cell counting. The results indicated that more monocytes and fewer neutrophils migrated into the lower chamber with A549-ORF7 cells than with control A549 cells (all $P<0.01$; Figures 3(e) and 3(f)). These results indicated that ORF7 attracted monocytes and repelled neutrophils in vitro.

\section{Discussion}

Previous postmortem examinations indicated that the lungs of COVID-19 patients, particularly the immune microenvironment, had significant alterations. These changes included alterations in T cells, B cells, macrophages, and neutrophils $[39,40]$. Lymphocytes, T cells, and B cells were less abun- dant and scattered in the lungs of these patients [30, 32, $39,41]$, but there was increased infiltration by monocytes, macrophages, and neutrophils [42]. After infection of the lungs, macrophages and neutrophils function as the first defense of the innate immune system, and these cells phagocytize pathogens and produce cytokines and chemokines that attract other immune cells [29]. The early recruitment of immune cells determines the local immune response and can even cause more widespread inflammation, such as a cytokine storm. Our present work examined the influence of ORF7 of SARS-CoV-2 on innate immunity. Our major result is that expression of ORF7 in type II pneumocytes (A549 cells) increased the level of CCL2, decreased the level of IL-8, and increased the migration of primary monocytes but decreased the migration of neutrophils in vitro.

The ORF7 gene is located in a region of the genomes of the SARS-CoV-2, SARS-CoV-1, and MERS viruses that have a high frequency of mutations [1]. Our results indicated that ORF7 has a specific function in the immune response to coronavirus infection. Monocytes produce IL-1, IL-6, IL18 , IL-33, TNF- $\alpha$, CCLs, and VEGF, and these molecules have critical roles in cytokine release and recruitment of other immune cells [43]. IL-6 and IL-1 are proinflammatory cytokines and the predominant inducers of the cytokine storm $[43,44]$. Additionally, IL- 6 can activate macrophages, which produce more cytokines and chemokines [43]. Neutrophils are also produced early in response to infection, and neutrophil chemotaxis in humans is usually mediated by factors such as IL-8, IL- 1 , TNF- $\alpha$, and complement C5a [45]. Neutrophils, like macrophages, produce a range of cytokines (TNF- $\alpha$, ILs, GCSF, MCSF, and GMCSF) and chemokines (IL-8, CXCL10, CXCL9, CCL2, CCL3, and CCL4) $[43,46]$. Thus, neutrophils have direct antipathogen effects (phagocytosis) and indirect antipathogen effects (stimulation by cytokines). Macrophages and neutrophils thus play critical roles during the acute phase of pneumonia following viral infection. 
Patients with COVID-19 have greater levels of peripheral monocytes than healthy controls $[47,48]$. Recent evidence showed massive macrophage infiltration of the lungs of deceased COVID-19 patients, including intra-alveolar CD68+ macrophages [30, 31, 49]. The monocytes in these tissues (macrophages) may be at a stage of active proliferation in the lung alveolar spaces of these patients [50].

Previous research reported that the blood neutrophil count of SARS patients was associated with the severity of their pneumonia [51]. In contrast, other studies reported that the blood neutrophil count of COVID-19 patients was inversely associated with disease severity [47, 52, 53]. Although virus infections, such as influenza, induce neutrophil infiltration in the respiratory tract, the status of neutrophils in the lungs of COVID-19 patients appears paradoxical $[30-33,54,55]$. Several autopsy reports found no neutrophil infiltration in the lungs of COVID-19 patients, but there was neutrophil infiltration of the liver [31]. Some reports that found minor infiltrations of neutrophils in the lungs attributed this to secondary infections [33]. A report of 4 patients with COVID-19 found that only 1 patient had neutrophil infiltration of the lungs [55]. Another report of two cases found neutrophil infiltration in 1 patient's pulmonary interstitium [30]. Other studies reported the presence of megakaryocytes, dendritic cells, and natural killer cells in the lungs of deceased COVID-19 patients [33, 40].

The present in vitro study found that overexpression of the SARS-CoV-2 ORF7 in cultured type II alveolar cells (A549) upregulated the expression of CCL2, a chemokine that functions in monocyte chemotaxis. This suggests that ORF7 may accelerate the progression of local inflammation after viral infection. Our in vitro studies also found that ORF7 downregulated the expression of IL-8. This suggests that ORF7 may block the migration of neutrophils. Greater neutrophil infiltration of the lungs could exacerbate the cytokine storm and worsen the patient's condition due to lymphopenia [56].

There is evidence that a specific variant of SARS-CoV-2 which has mutation N501Y in the S protein and was first reported in London [57] is now widespread. Because this mutation is in the receptor-binding domain of the $S$ protein, this variant likely has altered binding capacity to its ACE2 receptor. Even though this mutation was in the $S$ protein, recent research reported the efficacy of neutralizing antibodies in mice [58]. It is important to consider that the mutation frequency of RNA viruses, such as coronaviruses and influenza viruses, is higher than that of DNA viruses.

Our study indicated that ORF7 of SARS-CoV-2 had specific effects on the immunological changes. ORF7 may therefore contribute to the unique immunological profile of the lung tissues of patients with COVID-19, although the functions of other ORFs should also be examined. ORF7 can be the target for the development of antiCOVID-19 drugs in the future. The inflammation environment in the lung is one of the major risks for severe COVID-19 patients. In addition, ORF7 and other genes, including the structure and nonstructure proteins, combinatorially involve in the inflammatory environment in the lung after the infection.
However, there are limitations to our work. Many other physical and pathological factors impact the inflammation status of the lungs of patients with SARS-CoV-2 infections. For example, the genomic RNA sensor system triggers the innate immune reaction, stimulates the translation of other proteins from the genome or subgenome of the virus, and may thereby increase lung injury. Consequently, appropriated animal models can be employed for the determination of ORF7 in vivo in the future. Additionally, the effect of ORF7 on the microenvironment of the lung should not be considered alone. Instead, there should be a systematic examination of the impact of all factors and their interactions on lung inflammation.

\section{Data Availability}

There are no publicly archived datasets analysed in this study.

\section{Ethical Approval}

Our study was approved by the Institutional Ethics Committee of the First Affiliated Hospital of Zhejiang University.

\section{Consent}

Written informed consent of the legal guardian was obtained.

\section{Conflicts of Interest}

The authors declare no conflict of interest.

\section{Authors' Contributions}

Z.C. and H.Z. are responsible for the conceptualization; G.W., J.G., F.W., C.H., J.S., L.X., Z.G., and Q.Z. for the methodology; G.W. and J.G. for the formal analysis; G.W. and J.G. for the data curation; G.W. and J.G. for writing original draft preparation; Z.C., H.Z., and G.L. for writing-review and editing; Z.C. and H.Z. for the supervision; Z.C. for the project administration; and Z.C. for the funding acquisition, and Gang Wang and Jun Guan contributed equally to this work.

\section{Acknowledgments}

This research was funded by the National Science and Technology Major Project of China (2018ZX10302206003, 2017ZX10202203-007, and 2017ZX10202203-008), Zhejiang Health and Family Planning Research Projects (2016KYA162 and 2015KYA046), and Major Science and Technology Projects for Liver Disease Research Fund of Ningbo.

\section{References}

[1] P. Zhou, X.-L. Yang, X.-G. Wang et al., “Addendum: A pneumonia outbreak associated with a new coronavirus of probable bat origin," Nature, vol. 588, no. 7836, p. E6, 2020. 
[2] J. Bedford, D. Enria, J. Giesecke et al., "COVID-19: towards controlling of a pandemic," Lancet, vol. 395, no. 10229, pp. 1015-1018, 2020.

[3] J. Cao, W. J. Tu, W. Cheng et al., "Clinical features and shortterm outcomes of 102 patients with coronavirus disease 2019 in Wuhan, China," Clinical Infectious Diseases, vol. 71, no. 15, pp. 748-755, 2020.

[4] Z. Zhu, X. Lian, X. Su, W. Wu, G. A. Marraro, and Y. Zeng, "From SARS and MERS to COVID-19: a brief summary and comparison of severe acute respiratory infections caused by three highly pathogenic human coronaviruses," Respir Res, vol. 21, no. 1, p. 224, 2020.

[5] M. D. Christian, S. M. Poutanen, M. R. Loutfy, M. P. Muller, and D. E. Low, "Severe acute respiratory syndrome," Clinical Infectious Diseases, vol. 38, no. 10, pp. 1420-1427, 2004.

[6] A. Al-Omari, A. A. Rabaan, S. Salih, J. A. Al-Tawfiq, and Z. A. Memish, "MERS coronavirus outbreak: implications for emerging viral infections," Diagnostic Microbiology and Infectious Disease, vol. 93, no. 3, pp. 265-285, 2019.

[7] F. Krammer, "SARS-CoV-2 vaccines in development," Nature, vol. 586, no. 7830, pp. 516-527, 2020.

[8] Y. Dong, T. Dai, Y. Wei, L. Zhang, M. Zheng, and F. Zhou, "A systematic review of SARS-CoV-2 vaccine candidates," Signal Transduct Target Ther, vol. 5, no. 1, p. 237, 2020.

[9] R. Yan, Y. Zhang, Y. Li, L. Xia, Y. Guo, and Q. Zhou, "Structural basis for the recognition of SARS-CoV-2 by full-length human ACE2," Science, vol. 367, no. 6485, pp. 1444-1448, 2020.

[10] Y. Wu, F. Wang, C. Shen et al., "A noncompeting pair of human neutralizing antibodies block COVID-19 virus binding to its receptor ACE2," American Association for the Advancement of Science, vol. 368, no. 6496, pp. 1274-1278, 2020.

[11] J. Lan, J. Ge, J. Yu et al., "Structure of the SARS-CoV-2 spike receptor-binding domain bound to the ACE2 receptor," Nature, vol. 581, no. 7807, pp. 215-220, 2020.

[12] X. Y. Ge, J. L. Li, X. L. Yang et al., "Isolation and characterization of a bat SARS-like coronavirus that uses the ACE2 receptor," Nature, vol. 503, no. 7477, pp. 535-538, 2013

[13] S. M. Khade, S. M. Yabaji, and J. Srivastava, "An update on COVID-19: SARS-CoV-2 life cycle, immunopathology, and BCG vaccination," Preparative Biochemistry \& Biotechnology, vol. 51, no. 7, pp. 650-658, 2021.

[14] P. S. Masters, "The molecular biology of coronaviruses," Advances in Virus Research, vol. 66, pp. 193-292, 2006

[15] P. V'Kovski, A. Kratzel, S. Steiner, H. Stalder, and V. Thiel, "Coronavirus biology and replication: implications for SARSCoV-2," Nature Reviews. Microbiology, vol. 19, no. 3, pp. 155-170, 2021.

[16] R. A. Khailany, M. Safdar, and M. Ozaslan, "Genomic characterization of a novel SARS-CoV-2," Gene Rep, vol. 19, p. $100682,2020$.

[17] N. Vabret and J. M. Blander, "Sensing microbial RNA in the cytosol," Frontiers in Immunology, vol. 4, p. 468, 2013

[18] J. Y. Li, C. H. Liao, Q. Wang et al., "The ORF6, ORF8 and nucleocapsid proteins of SARS-CoV-2 inhibit type I interferon signaling pathway," Virus Research, vol. 286, p. 198074, 2020.

[19] Y. Ren, T. Shu, D. Wu et al., "The ORF3a protein of SARSCoV-2 induces apoptosis in cells," Cellular \& Molecular Immunology, vol. 17, no. 8, pp. 881-883, 2020.

[20] X. Liu, W. Song, X. Zhang et al., "Downregulating LncRNA XIST attenuated contrast-induced nephropathy injury via reg- ulating miR-133a-3p/NLRP3 axis," Journal of Thrombosis and Thrombolysis, 2021.

[21] R. Corriden, A. Moshensky, C. M. Bojanowski et al., "E-cigarette use increases susceptibility to bacterial infection by impairment of human neutrophil chemotaxis, phagocytosis, and NET formation," American Journal of Physiology. Cell Physiology, vol. 318, no. 1, pp. C205-c214, 2020.

[22] B. Xu, A. Janicova, J. T. Vollrath et al., "Club cell protein 16 in sera from trauma patients modulates neutrophil migration and functionality via CXCR1 and CXCR2," Mol Med, vol. 25, no. 1, p. 45, 2019.

[23] B. Bonilauri, M. D. M. Santos, A. C. Camillo-Andrade et al., "The impact of blood-processing time on the proteome of human peripheral blood mononuclear cells," Biochim Biophys Acta Proteins Proteom, vol. 1869, no. 3, article 140581, 2020.

[24] S. Vitale, A. Schmid-Alliana, V. Breuil et al., "Soluble fractalkine prevents monocyte chemoattractant protein-1-induced monocyte migration via inhibition of stress-activated protein kinase 2/p38 and matrix metalloproteinase activities," Journal of Immunology, vol. 172, no. 1, pp. 585-592, 2004.

[25] I. Bournazou, J. D. Pound, R. Duffin et al., “Apoptotic human cells inhibit migration of granulocytes via release of lactoferrin," The Journal of Clinical Investigation, vol. 119, no. 1, pp. 20-32, 2009.

[26] L. Saraiva, L. Wang, M. Kammel et al., "Comparison of volumetric and bead-based counting of CD34 cells by singleplatform flow cytometry," Cytometry. Part B, Clinical Cytometry, vol. 96, no. 6, pp. 508-513, 2019.

[27] M. Hoffmann, H. Kleine-Weber, S. Schroeder et al., "SARSCoV-2 cell entry depends on ACE2 and TMPRSS2 and is blocked by a clinically proven protease inhibitor," Cell, vol. 181, no. 2, pp. 271-280.e8, 2020.

[28] J. R. Cooper, M. B. Abdullatif, E. C. Burnett et al., "Long term culture of the A549 cancer cell line promotes multilamellar body formation and differentiation towards an alveolar type II pneumocyte phenotype," PLoS One, vol. 11, no. 10, article e0164438, 2016.

[29] M. Galeas-Pena, N. McLaughlin, and D. Pociask, "The role of the innate immune system on pulmonary infections," Biological Chemistry, vol. 400, no. 4, pp. 443-456, 2019.

[30] S. Li, L. Jiang, X. Li et al., "Clinical and pathological investigation of patients with severe COVID-19," JCI Insight, vol. 5, no. $12,2020$.

[31] X.-W. Bian, "Autopsy of COVID-19 patients in China," National Science Review, vol. 7, no. 9, pp. 1414-1418, 2020.

[32] L. M. Barton, E. J. Duval, E. Stroberg, S. Ghosh, and S. Mukhopadhyay, "Covid-19 autopsies, Oklahoma, USA," American Journal of Clinical Pathology, vol. 153, no. 6, pp. 725-733, 2020.

[33] S. E. Fox, A. Akmatbekov, J. L. Harbert, G. Li, J. Quincy Brown, and R. S. Vander Heide, "Pulmonary and cardiac pathology in African American patients with COVID-19: an autopsy series from New Orleans," The Lancet Respiratory Medicine, vol. 8, no. 7, pp. 681-686, 2020.

[34] G. Arango Duque and A. Descoteaux, "Macrophage cytokines: involvement in immunity and infectious diseases," Frontiers in Immunology, vol. 5, p. 491, 2014.

[35] M. E. Hammond, G. R. Lapointe, P. H. Feucht et al., "IL-8 induces neutrophil chemotaxis predominantly via type I IL-8 receptors," Journal of Immunology, vol. 155, no. 3, pp. 1428$1433,1995$. 
[36] M. Gschwandtner, R. Derler, and K. S. Midwood, "More than just attractive: how CCL2 influences myeloid cell behavior beyond chemotaxis," Frontiers in Immunology, vol. 10, p. 2759, 2019.

[37] Z. Liu, C. Wu, Y. Pan et al., "NDR2 promotes the antiviral immune response via facilitating TRIM25-mediated RIG-I activation in macrophages," Sci $A d v$, vol. 5, no. 2, article eaav0163, 2019.

[38] R. Singh, T. Hui, A. Matsui et al., "Modulation of infectionmediated migration of neutrophils and CXCR2 trafficking by osteopontin," Immunology, vol. 150, no. 1, pp. 74-86, 2017.

[39] D. Wichmann, J. P. Sperhake, M. Lutgehetmann et al., "Autopsy findings and venous thromboembolism in patients with COVID-19," Annals of Internal Medicine, vol. 173, no. 4, pp. 268-277, 2020.

[40] Y. Zhang, Y. Gao, L. Qiao, W. Wang, and D. Chen, "Inflammatory response cells during acute respiratory distress syndrome in patients with coronavirus disease 2019 (COVID-19)," Annals of Internal Medicine, vol. 173, no. 5, pp. 402-404, 2020.

[41] A. V. Rapkiewicz, X. Mai, S. E. Carsons et al., "Megakaryocytes and platelet-fibrin thrombi characterize multi-organ thrombosis at autopsy in COVID-19: a case series," EClinicalMedicine, vol. 24, p. 100434, 2020.

[42] A. A. Stegelmeier, J. P. van Vloten, R. C. Mould et al., "Myeloid cells during viral infections and inflammation," Viruses, vol. 11, no. 2, p. 168, 2019.

[43] D. C. Fajgenbaum and C. H. June, "Cytokine storm," The New England Journal of Medicine, vol. 383, no. 23, pp. 2255-2273, 2020.

[44] W. J. Tu, J. Cao, L. Yu, X. Hu, and Q. Liu, "Clinicolaboratory study of 25 fatal cases of COVID-19 in Wuhan," Intensive Care Medicine, vol. 46, no. 6, pp. 1117-1120, 2020.

[45] C. J. Thomas and K. Schroder, "Pattern recognition receptor function in neutrophils," Trends in Immunology, vol. 34, no. 7, pp. 317-328, 2013.

[46] M. A. Cassatella, "Neutrophil-derived proteins: selling cytokines by the pound," Advances in Immunology, vol. 73, pp. 369-509, 1999.

[47] R. Wang, M. Pan, X. Zhang et al., "Epidemiological and clinical features of 125 hospitalized patients with COVID-19 in Fuyang, Anhui, China," International Journal of Infectious Diseases, vol. 95, pp. 421-428, 2020.

[48] L. Wang, Y. Duan, W. Zhang et al., "Epidemiologic and clinical characteristics of 26 cases of COVID-19 arising from patientto-patient transmission in Liaocheng, China," Clinical Epidemiology, vol. Volume 12, pp. 387-391, 2020.

[49] T. Adachi, J. M. Chong, N. Nakajima et al., "Clinicopathologic and immunohistochemical findings from autopsy of patient with COVID-19, Japan," Emerg Infect Dis, vol. 26, no. 9, pp. 2157-2161, 2020.

[50] S. F. Lax, K. Skok, P. Zechner et al., "Pulmonary arterial thrombosis in COVID-19 with fatal outcome: results from a prospective, single-center, clinicopathologic case series," Annals of Internal Medicine, vol. 173, no. 5, pp. 350-361, 2020.

[51] R. S. Wong, A. Wu, K. F. To et al., "Haematological manifestations in patients with severe acute respiratory syndrome: retrospective analysis," BMJ, vol. 326, no. 7403, pp. 1358-1362, 2003.

[52] G. Zhang, C. Hu, L. Luo et al., "Clinical features and shortterm outcomes of 221 patients with COVID-19 in Wuhan, China," Journal of Clinical Virology, vol. 127, p. 104364, 2020.
[53] W. Yang, Q. Cao, L. Qin et al., "Clinical characteristics and imaging manifestations of the 2019 novel coronavirus disease (COVID-19):A multi-center study in Wenzhou city, Zhejiang, China," The Journal of Infection, vol. 80, no. 4, pp. 388-393, 2020.

[54] T. Watanabe, J. Tisoncik-Go, N. Tchitchek et al., "1918 influenza virus hemagglutinin (HA) and the viral RNA polymerase complex enhance viral pathogenicity, but only HA induces aberrant host responses in mice," Journal of Virology, vol. 87, no. 9, pp. 5239-5254, 2013.

[55] S. Tian, Y. Xiong, H. Liu et al., "Pathological study of the 2019 novel coronavirus disease (COVID-19) through postmortem core biopsies," Modern Pathology, vol. 33, no. 6, pp. 1007$1014,2020$.

[56] A. Tufan, A. Avanoglu Guler, and M. Matucci-Cerinic, "COVID-19, immune system response, hyperinflammation and repurposing antirheumatic drugs," Turk J Med Sci, vol. 50, no. SI-1, pp. 620-632, 2020.

[57] J. Wise, "Covid-19: new coronavirus variant is identified in UK,” BMJ, vol. 371, p. m4857, 2020.

[58] H. Gu, Q. Chen, G. Yang et al., "Adaptation of SARS-CoV-2 in BALB/c mice for testing vaccine efficacy," Science, vol. 369, no. 6511, pp. 1603-1607, 2020. 